\title{
Analysis of hybrid viscous damper by real time hybrid simulations
}

\author{
Mark Laier Brodersen ${ }^{\mathrm{a}, \mathrm{b}, *}, \mathrm{Ge}_{\mathrm{Ou}}^{\mathrm{c}}$, Jan Høgsberg ${ }^{\mathrm{a}}$, Shirley Dyke ${ }^{\mathrm{d}}$ \\ ${ }^{a}$ Department of Mechanical Engineering, Technical University of Denmark, DK-2800 \\ Kgs. Lyngby, Denmark \\ ${ }^{b}$ National Oilwell Varco Denmark I/S, Priorparken 480, DK-2605 Brndby, Denmark \\ ${ }^{c}$ Lyles School of Civil Engineering, Purdue University, West Lafayette, IN 47906 \\ ${ }^{d}$ School of Mechanical Engineering, Purdue University, West Lafayette, IN 47906
}

\begin{abstract}
Results from real time hybrid simulations are compared to full numerical simulations for a hybrid viscous damper, composed of a viscous dashpot in series with an active actuator and a load cell. By controlling the actuator displacement via filtered integral force feedback the damping performance of the hybrid viscous damper is improved, while for pure integral force feedback the damper stroke is instead increased. In the real time hybrid simulations viscous damping is emulated by a bang-bang controlled Magneto-Rheological (MR) damper. The controller activates high-frequency modes and generates drift in the actuator displacement, and only a fraction of the measured damper force can therefore be used as input to the investigated integral force feedback in the real time hybrid simulations.
\end{abstract}

Keywords: Hybrid control, real time hybrid simulation, Integral Force Feedback, MR damper, bang-bang controller

\section{Introduction}

Passive control systems for structural control will not necessarily be optimal for any type of loading [1], and its damping efficiency will also be fully dependent on the relative response of the structure at the position of the

\footnotetext{
*Corresponding author at: National Oilwell Varco Denmark I/S, Priorparken 480, DK2605 Brøndby, Denmark

Email address: MarkLaier.Brodersen@nov.com, (Mark Laier Brodersen)

Preprint submitted to Elsevier

June 30, 2016
}

(c) 2016. This manuscript version is made available under the Elsevier user license http://www.elsevier.com/open-access/userlicense/1.0/ 
damper, thereby limiting the effectiveness of a passive system [2]. Contrary to passive systems, active control systems require an external power source, which makes them more costly to operate and prone to power loss [3]. Furthermore, due to the nature of feedback an active control system can become unstable, which again can lead to a loss of structural integrity in the main structure. In semi-active damping the damper properties may be controlled instantaneously by modern optimal control strategies [4] to improve performance without the potential instabilities often associated with fully active control. The magneto-rheological (MR) damper is widely applied in semiactive control, as it can be controlled accurately and robustly in real-time $[4,5]$ and even realize active forces with apparent negative stiffness when controlled properly, as demonstrated in [6]. A comparison of semi-active control strategies for MR dampers is provided in [7].

An alternative approach to overcome some of the drawbacks of passive and active control systems is the so-called hybrid damper concept, where hybrid refers to the combined use of active and passive control, in which the properties of the two systems are merged in a favorable way [8]. For example, hybrid or passive-active concepts are employed in [9] to effectively damp multiple modes by a pendulum vibration absorber or to realize effective damping by sky-hook control forces with absolute structure motion as sensor input [10]. The hybrid viscous damper concept introduced in [11] is a novel damper concept for active control of large structures. The concept consists of a passive viscous dash-pot in series with an active actuator and a load cell unit for measuring the damper force, and with the actuator motion regulated by a decentralized collocated control algorithm based on feedback from the load cell. When the actuator motion is controlled using a filtered Integral Force Feedback (IFF) scheme as in [11], the hybrid damper introduces a phase lead between damper force and damper velocity, which leads to increased attainable damping. When instead the filter time constant is set to zero, as in [12], a pure IFF scheme is recovered [13]. The hybrid damper then performs like a viscous damper with the force fully in phase with velocity, but also with the ability to increase the amplitude of the displacement over the viscous dash-pot. This stroke amplification property may increase the feasibility of installing the hybrid viscous damper in a flexible structure at a location with inherently small deformations. The numerical simulations conducted in $[11,12]$ demonstrate the large potential and the performance of the hybrid viscous damper concept, which motivates further analysis of the concept. Thus, as the next step the performance of the hybrid damper 
should be investigated experimentally to verify the concept and in particular to investigate the influence of the drift in the actuator force observed in [12].

The aim of the present paper is to perform an experimental verification of the performance of the hybrid viscous damper concept. The analysis is performed by comparing numerical results equivalent to the results in $[11,12]$ with experimental results from real time hybrid simulations (RTHS) performed at the Intelligent Infrastructure Systems Lab at Purdue University. RTHS is a testing technique in which the structural system is divided into an experimental substructure and a numerical substructure, which are tested together as a single system in real time $[14,15]$. Since the test is executed in real time it allows for physical testing of the dampers and dissipative devices in connection with a numerical model of a primary structure.

In this paper an experimental substructure representing a hybrid viscous damper is tested together with a numerical shear frame model in order to validate the results presented in [11], and subsequently with a wind turbine model in order to compare with the results presented in [12]. Initially, for completeness the hybrid viscous damper concept is briefly presented in Section 2, which also contains a summary of the two force feedback control strategies. In Section 3 the experimental setup used for the RTHS and the particular partitioning into an experimental substructure and a numerical substructure is explained. The experimental substructure represents a model of the hybrid damper concept, and consist of a magnetorheogical (MR) damper in series with a hydraulic actuator and a load cell. An MR damper is used since ideal viscous damping is difficult to achieve by passive means. However, as the hybrid viscous damper is based on linear control theory the damper element in series with the actuator must be linear or linearized. The inherently nonlinear MR damper used in the experimental setup is therefore controlled by a bang-bang controller to emulate the behavior of an ideal linear viscous dash-pot. Furthermore, to avoid instabilities due to the inherent phase lags and time delays in RTHS and the corresponding measurement noise, only part of the damper force is measured in the experimental substructure, while the remaining part is directly represented in the numerical substructure, also containing the shear frame or wind turbine structure. Thus, in Section 4 the numerical results for the hybrid viscous damper concepts are only verified by partial real time hybrid simulations, and special attention is therefore paid to the performance of the bang-bang controller and its influence on the overall damping performance, the drift of the actuator displacement and the fraction of experimental damper force represented in the RTHS. Finally, in 
Section 5 the results and findings of the present analysis are summarized and discussed.

\section{Hybrid damper concept}

The hybrid damper concept is illustrated in figure 1. The hybrid damper consists of a dash-pot with viscous coefficient $c$ in series with a load cell and an active actuator with controllable piston motion $q(t)$. The full displacement across the terminals of the hybrid damper is denoted by $u(t)$, whereby the damper force $f(t)$ is given as

$$
f(t)=c(\dot{u}(t)-\dot{q}(t))
$$

where the dot denotes time differentiation. The properties of the hybrid viscous damper are conveniently investigated in the frequency domain, which is obtained by assuming the following complex harmonic solutions

$$
u(t)=\bar{u} \exp (i \omega t) \quad, \quad q(t)=\bar{q} \exp (i \omega t) \quad, \quad f(t)=\bar{f} \exp (i \omega t)
$$

where $\omega$ is the angular frequency and $i=\sqrt{-1}$ is the imaginary unit, while the bar denotes harmonic amplitudes. By substitution of (2) the harmonic amplitude of the damper force in (1) can be written as

$$
\bar{f}=i \omega c(\bar{u}-\bar{q})
$$

The damper force is in figure 1 measured by the load cell and fed back to control the actuator displacement in accordance with a particular control law, which consequently governs the overall performance and efficiency of the hybrid viscous damper.

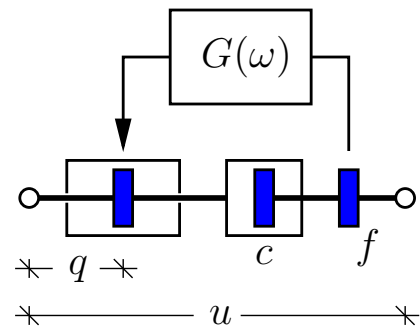

Figure 1: Hybrid viscous damper with integral force feedback. 


\subsection{Filtered integral force feedback}

It is demonstrated in [11] that effective structural damping is obtained when the velocity $\dot{q}(t)$ of the actuator piston motion is controlled by the following filtered Integral Force Feedback (IFF) equation,

$$
\tau \ddot{q}(t)+\dot{q}(t)=-g f(t)
$$

where $g$ is the control gain and $\tau \geq 0$ is the filter time scale. In the case of $g=0$ the passive viscous case associated with $\dot{q}(t)=0$ is recovered. By substitution of the harmonic representations in (2) the filtered IFF equation (4) can be written in the compact form $\bar{q}=G(\omega) \bar{f}$ with the frequency dependent control function

$$
G(\omega)=\frac{-g}{1+i \omega(i \omega \tau)}
$$

contained in the feedback loop in figure 1. Elimination of $\bar{q}$ in (3) by $\bar{q}=$ $G(\omega) \bar{f}$ determines the resulting force-displacement relation

$$
\frac{\bar{f}}{\bar{u}}=i \omega c H(\omega)
$$

introducing the normalized transfer function

$$
H(\omega)=\frac{1+i \omega \tau}{1-\nu+i \omega \tau} \quad, \quad \nu=c g
$$

with non-dimensional control gain $\nu$. In (6) the transfer function $H(\omega)$ represents the modification of the damper force relative to the pure viscous case obtained by $H(\omega)=1$. The real and the imaginary part of $H(\omega)$ are given as

$$
\operatorname{Re}[H(\omega)]=\frac{1-\nu+(\omega \tau)^{2}}{(1-\nu)^{2}+(\omega \tau)^{2}} \quad, \quad \operatorname{Im}[H(\omega)]=\frac{-\nu \omega \tau}{(1-\nu)^{2}+(\omega \tau)^{2}}
$$

Energy dissipation by the damper force in (6) is ensured by a positive imaginary part, represented by $\operatorname{Im}[\bar{f} / \bar{u}]>0$. With respect to the damper function $H(\omega)$ defined in (7) this dissipation condition is equivalent to a positive real part $(\operatorname{Re}[H(\omega)]>0)$ and therefore secured over the entire frequency domain by a control gain $\nu<1$. 

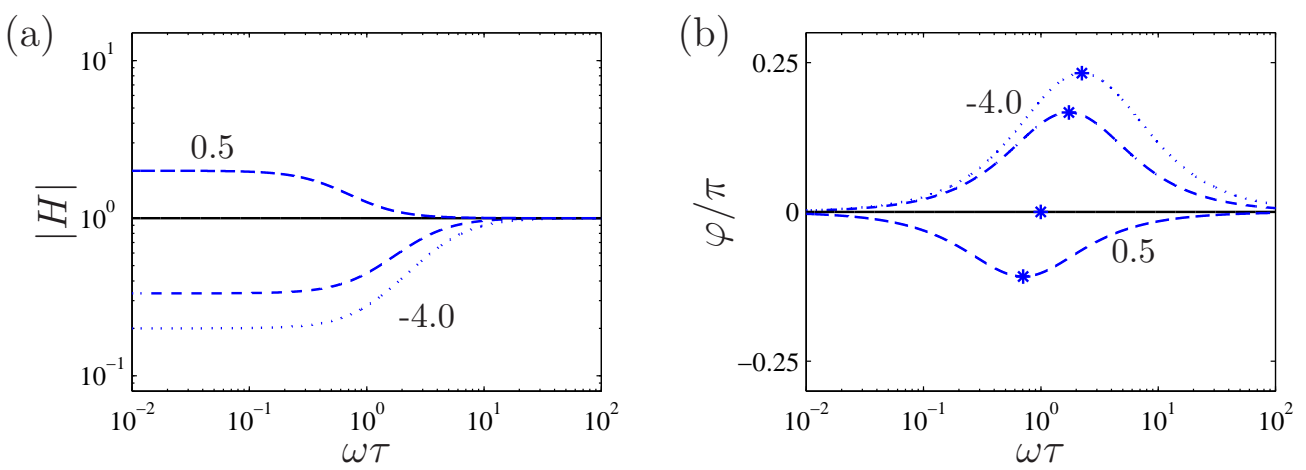

Figure 2: (a) Magnitude and (b) phase of the transfer function $H(\omega)$ for $\nu=0.5(--), 0.0(-),-2.0(-\cdot-)$ and $-4.0(\cdot \cdot)$.

\subsection{Increasing attainable damping}

As demonstrated in [11] the attainable damping can be increased compared to the pure viscous damper by adjusting the time scale $\tau$ so that the damper force component $\bar{f}$ operates ahead of the corresponding velocity component in a phase vector diagram. Figure 2 shows the magnitude $|H(\omega)|$ of the complex damper function and the corresponding phase angle $\varphi$, governed by the relation $\tan \varphi=\operatorname{Im}[H(\omega)] / \operatorname{Re}[H(\omega)]$. The individual curves in the figure represent the various non-dimensional gain values: $\nu=0.5(--), 0.0$ $(-),-2.0(-\cdot-)$ and $-4.0(\cdot)$. A positive phase angle $\varphi>0$ is associated with the desired phase lead of the damper force, and it follows from [11] and figure 2 that this is obtained by negative gains $(\nu<0)$. Furthermore, the extremum of the phase angle can be determined by the gradient condition $d \tan (\varphi) / d(\omega \tau)=0$, which gives $(\omega \tau)^{2}=1-\nu$. Thus, the time scale is determined as

$$
\tau=\frac{\sqrt{1-\nu}}{\omega_{s}}
$$

with respect to the natural frequency $\omega_{s}$ of the targeted vibration mode. The phase angle associated with $\tau$ determined in (9) is represented by the asterisks in figure 2 . The improvement of damper efficiency by an apparent negative damper stiffness is further discussed with respect to linear control strategies in [16].

A limitation associated with the filtered IFF equation in (4) is related to drift in the actuator, as discussed in [12]. This occurs because the control 
equation governs the actuator velocity $\dot{q}$, whereby the corresponding feedback relation (5) has an inherent pole at $s=i \omega=0$, potentially creating an artificial quasi-static contribution to $q$. To avoid drift by this quasi-static contribution the control equation (4) is extended to a full second order differential equation

$$
\tau \ddot{q}(t)+\left(1+\omega_{q} \tau\right) \dot{q}(t)+\omega_{q} q(t)=-g f(t)
$$

in which the corner frequency $\omega_{q}$ should be chosen sufficiently large to prevent drift, without severely deteriorating the overall damping performance of the hybrid viscous damper. A main goal of the RTHS considered in the following sections is to assess the influence of $\omega_{q}$ on both actuator drift and overall damper performance.

\subsection{Increasing damper stroke}

Alternatively, the integral force feedback strategy can be used within the hybrid viscous damper concept to effectively amplify the stroke $(u-q)$ across the viscous damper. As demonstrated in [12] the magnitude of the damper function directly represents the relative stroke,

$$
\left|\frac{\bar{u}-\bar{q}}{\bar{u}}\right|=|H(\omega)|
$$

and in the present section the damper parameters are therefore optimized with respect to maximizing the damper stroke factor $|H(\omega)|$.

For vanishing time scale $(\tau=0)$ in $(4)$ the pure IFF control scheme is recovered as

$$
\dot{q}(t)=-g f(t)
$$

By substitution of the harmonic representations in (2) the corresponding frequency domain expression $(i \omega \bar{q}=-g \bar{f})$ is obtained. The damper force relation in (6) is then determined by subsequent elimination of the actuator amplitude $\bar{q}$, identifying the frequency transfer function as

$$
H(\omega)=\frac{1}{1-\nu}
$$

where $\nu<1$ represents the stability condition. In this case $H(\omega)$ is realvalued and the hybrid damper therefore operates as a pure viscous damper with $1 /(1-\nu)$ representing the amplification factor defined in (11). Thus, 
the stroke across the viscous dashpot is amplified by positive non-dimensional gain values in the interval $0<\nu<1$. This demonstrates that the hybrid damper can be used to improve the feasibility of installing viscous dampers at locations in a flexible structure where the deformations are inherently small.

As for the filtered IFF scheme in (4) the pure IFF scheme in (12) is prone to drift in the actuator displacement. A augmentation to a full second order filter with filter frequency $\omega_{f}$ and filter time scale $\tau_{f}$ has therefore been proposed in [12],

$$
\tau_{f} \ddot{q}(t)+\dot{q}(t)+\omega_{f} q(t)=-g f(t)
$$

The corresponding frequency domain representation is obtained by substitution of (2), followed by elimination of $\bar{q}$ via the expression in (3). The damper force relation in (6) then defines the following transfer function associated with the modified control equation (14),

$$
H(\omega)=\frac{\omega_{f}-\tau_{f} \omega^{2}+i \omega}{\omega_{f}-\tau_{f} \omega^{2}+i \omega(1-\nu)}
$$

The magnitude of this complex-valued function is given as

$$
|H(\omega)|=\sqrt{\frac{\left(\omega_{f}-\tau_{f} \omega^{2}\right)^{2}+\omega^{2}}{\left(\omega_{f}-\tau_{f} \omega^{2}\right)^{2}+\omega^{2}(1-\nu)^{2}}}
$$

while the phase angle $\varphi$ is determined by the relation

$$
\tan \varphi=\frac{\operatorname{Im}[H(\omega)]}{\operatorname{Re}[H(\omega)]}=\frac{\omega \nu\left(\omega_{f}-\tau_{f} \omega^{2}\right)}{\omega^{2}(1-\nu)+\left(\omega_{f}-\tau_{f} \omega^{2}\right)^{2}}
$$

To maximize the amplification of the damper stroke, represented by the magnitude in (16), the filter frequency should be chosen as

$$
\omega_{f}=\tau_{f} \omega_{s}^{2}
$$

where $\omega_{s}$ is again the natural frequency of the dominant structural vibration mode. Substitution of (18) into both (16) and (17) gives the following expressions for the stroke amplification factor and the phase angle when $\omega=\omega_{s}$,

$$
\left|H\left(\omega_{s}\right)\right|=\left|\frac{1}{1-\nu}\right| \quad, \quad \varphi=0
$$

Thus, the second order filter equation in (14) recovers pure viscous damping $(\varphi=0)$ and a stroke amplification factor $\left|H\left(\omega_{s}\right)\right|$ that is equivalent to that for 
the pure IFF in (13). Thus, by carefully balancing the filter parameters $\omega_{f}$ and $\tau_{f}$ according to (18), the filtered integration format in (14) can be used to avoid drift in the actuator signal, without affecting the desired amplification properties of the hybrid viscous damper. This is also verified by the RTHS considered in the following sections.

From a design and calibration perspective the performance of the hybrid damper is mainly controlled by the values of the dimensionless gain $\nu$ and the corresponding filter time scale $\tau$. The differences between the various control regimes of the hybrid viscous damper and the corresponding system parameters $\nu$ and $\tau$ are summarized in table 1 .

Table 1: Overview of the two controllers

\begin{tabular}{|c|c|c|}
\hline & $0<\nu<1$ & $\nu<0$ \\
\hline$\tau>0$ & $\begin{array}{l}\text { FIFF controller with damper } \\
\text { force in phase lag. }\end{array}$ & $\begin{array}{l}\text { FIFF controller with damper } \\
\text { force in phase lead. } \\
\text { Validation case: } 10 \text { story shear } \\
\text { frame with top load. }\end{array}$ \\
\hline$\tau=0$ & $\begin{array}{l}\text { IFF controller with hybrid } \\
\text { damper stroke amplification. } \\
\text { Validation case: Offshore wind } \\
\text { turbine with wave load. }\end{array}$ & $\begin{array}{l}\text { IFF controller with hybrid } \\
\text { damper stroke reduction. }\end{array}$ \\
\hline
\end{tabular}

\section{Experimental setup}

Hybrid simulations constitute a test method that employs the technique of substructuring, in which the entire test structure is divided into two parts: the experimental substructure represented by a physical model in the laboratory and the numerical substructure represented by a numerical or mathematical model. This division implies that the physical tests conducted on the experimental substructure can be limited to the most critical structural components, which are typically of reduced size and with undefined structural properties.

A (quasi-static) hybrid simulation is performed by initially computing the displacement increment of the numerical substructure based on a suitable 
load increment. This computed displacement increment from the numerical substructure is then subsequently imposed on the experimental substructure by means of actuators at the interface between the two substructures. In the experimental substructure imposing this displacement generates restoring forces at the interface, which are measured by load cells and used to update the state of the numerical substructure. This sequence of steps is continued in an iterative procedure until the two substructures are in equilibrium with each other, whereafter the system is ready to be advanced by a new load increment.

In real time hybrid simulation (RTHS) the quasi-static process described above is simply conducted in real time, thereby allowing for physical testing of rate dependent components of a structure, such as a damper (the experimental substructure) attached to a large primary structure (the numerical substructure) as described in [17, 7]. In the present investigation RTHS is applied for the analysis of a model of the hybrid viscous damper attached to two types of structures: A 10 story shear frame structure and an offshore wind turbine structure. Hereby, the present results obtained by RTHS are comparable to the numerical results previously obtained in [11] for the shear frame and in [12] for the offshore wind turbine. The hybrid damper model, consisting of a magnetorheogical (MR) damper in series with an actuator and a load cell, is considered as the experimental substructure, while the primary structure (shear frame or wind turbine) is represented by the numerical substructure. An overview of the two different experimental setups is provided in table 2. It shows that only a part of the measured damper force in the experimental substructure is used as input to the integral force feedback equation. Thus, the present paper considers partial RTHS to verify the performance of the hybrid viscous damper concepts.

\subsection{Numerical substructure}

The structure to be controlled is represented by a finite element (FE) model with mass matrix $\mathbf{M}$ and stiffness matrix $\mathbf{K}$, whereby the equation of motion can be written as

$$
\mathbf{M} \ddot{\mathbf{u}}(t)+\mathbf{K u}(t)=\mathbf{f}(t)-\mathbf{w} f(t)
$$

This system of equations is solved with respect to the displacement of the structural degrees of freedom contained in the vector $\mathbf{u}(t)$. The matrices $\mathbf{M}$ 
Table 2: Overview of the two experimental setups

\begin{tabular}{c|cc} 
& FIFF & IFF \\
\hline$\nu$ & $0,-2,-4$ & $0.5,0.75$ \\
$\tau$ & $(\sqrt{1-\nu}) / \omega_{1}$ & 0 \\
$\omega_{q}$ & $\omega_{1} / 5, \omega_{1} / 8, \omega_{1} / 20$ & Not applicable \\
$\omega_{f}=\tau_{f} \omega_{1}^{2}$ & Not applicable & $\omega_{1} / 5, \omega_{1} / 8$ \\
Numerical & 10 story shear frame, & Offshore wind turbine, \\
substructure & $50 \%$ viscous damper force & $90 \%$ viscous damper force \\
Experimental & $50 \%$ viscous damper force & $10 \%$ viscous damper force \\
substructure & emulated with MR damper, & emulated with MR damper, \\
& FIFF controller & IFF controller
\end{tabular}

and $\mathbf{K}$ are in the present two cases derived from both a numerical shear frame model and a realistic offshore wind turbine model. The time dependent vector $\mathbf{f}(t)$ represents the external loading, $\mathbf{w}$ is the connectivity vector defining the connection of the hybrid viscous damper to the structure, while $f(t)$ is the force of the hybrid viscous damper. Structural damping is omitted in the numerical model in (20) because the theoretical results in [11] are presented for an undamped structure, whereby the resulting damping of the full combined model may be entirely attributed to the performance of the hybrid viscous damper.

\subsubsection{Damper force partitioning}

To avoid instabilities in the RTHS, due to finite sampling rate and the inherent phase lags and time delays, the total damper force $f(t)$ is partitioned into two parts: the force $f_{e}(t)$ from the actual hybrid damper representing the experimental substructure and $f_{n}(t)$ from a numerical hybrid viscous damper model contained in the numerical substructure. Thus, the resulting damper force is given by the sum

$$
f(t)=f_{e}(t)+f_{n}(t)
$$

The ratio between the damper force from the experimental substructure and the damper force from the numerical substructure is governed by an interpolation parameter $\eta$, which simply determines the ratio between the viscous 
(a)

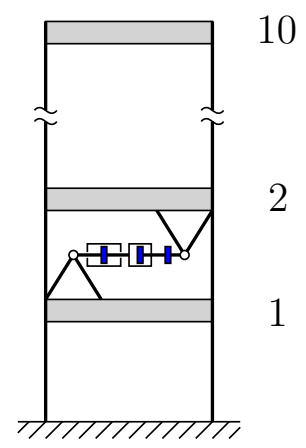

(b)

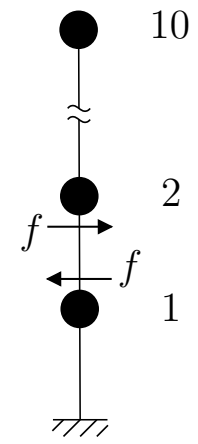

Figure 3: Diagram of (a) the Shear frame with hybrid damper and (b) corresponding FE model with damper force $f$

parameter $c_{e}$ realized by the controllable MR damper in the experimental substructure and the remaining viscous parameter $c_{n}$ in the numerical substructure. Thus, the split in (21) of the total damper force can instead be represented by the following split of the total viscous damping parameter $c_{\text {tot }}$,

$$
c_{e}=\eta c_{t o t} \quad, \quad c_{n}=(1-\eta) c_{t o t}
$$

By changing the value of $\eta$ between 0 and 1 the ratio between the damper force shifts between the case where the entire damper force is computed numerically $(\eta=0)$ and the situation where the damper force is fully represented in the experimental substructure $(\eta=1)$. As indicated in table 2 the interpolation parameter $\eta$ is substantially less than unity for both structures and the results presented in Section 4 are therefore only partially confirmed by the RTHS, as discussed further in Section 5 .

\subsubsection{Shear frame model}

The shear frame model is in the following used for analysis of the filtered IFF scheme described in Section 2.2 and the model is therefore chosen identical to the numerical shear frame model used in the background paper [11]. The model consist of ten stories with the damper connected between the first and second floor, as illustrated in figure 3(a). The shear frame is modeled by a simple 10 dof FE model with lumped masses, as illustrated in figure 3(b). 
Hereby, the mass matrix and the stiffness matrix are given as

$$
\mathbf{M}=m\left[\begin{array}{cccc}
1 & & & \\
& 1 & & \\
& & \ddots & \\
& & & 1
\end{array}\right] \quad, \quad \mathbf{K}=k\left[\begin{array}{cccc}
2 & -1 & & \\
-1 & 2 & & \\
& \ddots & -1 \\
& & -1 & 1
\end{array}\right]
$$

where the lumped mass $m$ represents the concentrated floor mass, while $k$ represents the resulting stiffness of the connecting walls. The model used in the RTHS is assumed to have mass $m=1 \mathrm{~kg}$ and stiffness $k=1.77$ $\mathrm{N} / \mathrm{m}$, which gives a lowest eigenfrequency of $\omega_{1} / 2 \pi=1 \mathrm{~Hz}$. The connectivity vector, which describes the connection of the hybrid viscous damper between the first and second floor, is given as

$$
\mathbf{w}=[-1,1,0,0,0,0,0,0,0,0]^{T}
$$

Because the hybrid viscous damper is a collocated damping device the deformation of the hybrid viscous damper is consequently given as $u(t)=\mathbf{w}^{T} \mathbf{u}(t)$. When inserting the damper force expression in (21) and the feedback relation in (10) into the structural equation of motion in (20) the governing closedloop equations for the structural degrees of freedom $\mathbf{u}(t)$ and the numerical actuator displacement $q(t)$ is given as

$$
\begin{aligned}
{\left[\begin{array}{ll}
\mathbf{M} & \mathbf{0} \\
\mathbf{0}^{T} & \tau
\end{array}\right]\left[\begin{array}{l}
\ddot{\mathbf{u}}(t) \\
\ddot{q}(t)
\end{array}\right]+} & +\left[\begin{array}{cc}
c_{n} \mathbf{w} \mathbf{w}^{T} & -c_{n} \mathbf{w} \\
\nu \mathbf{w}^{T} & 1-\nu+\omega_{q} \tau
\end{array}\right]\left[\begin{array}{c}
\dot{\mathbf{u}}(t) \\
\dot{q}(t)
\end{array}\right] \\
+ & {\left[\begin{array}{cc}
\mathbf{K} & \mathbf{0} \\
\mathbf{0}^{T} & \omega_{q}
\end{array}\right]\left[\begin{array}{l}
\mathbf{u}(t) \\
q(t)
\end{array}\right]=\left[\begin{array}{c}
\mathbf{f}(t)-\mathbf{w} f_{e}^{m} \\
0
\end{array}\right] }
\end{aligned}
$$

The optimum viscous damping parameter is determined for the first vibration mode of the structure by a root locus analysis [11], and the subsequent split of the optimal viscous parameter into an experimental parameter $c_{e}$ and a numerical parameter $c_{n}$ is then determined by (22) for a given interpolation parameter $\eta$. In (24) the superscript $m$ indicates that $f_{e}^{m}$ represents the damper force actually measured by the load cell, as discussed in further detail in Section 3.2.

The shear frame model is assumed to be loaded locally at the top floor in the numerical substructure by a linearly increasing force, which is then 
removed after time $t=2 T_{1}$, where $T_{1}=2 \pi / \omega_{1}$ is the vibration period of the first mode shape of the structure. The external load vector $\mathbf{f}(t)$ is therefore given as

$$
\mathbf{f}(t)=p(t)[0,0, \ldots, 0,1]^{T}
$$

where the load intensity $p(t)$ is described as

$$
\frac{p(t)}{p_{0}}= \begin{cases}t /\left(2 T_{1}\right) & , \quad t \leq 2 T_{1} \\ 0 & , \quad t>2 T_{1}\end{cases}
$$

with $p_{0}$ representing the maximum value of the load intensity. The ramp load in (26) is chosen because it first of all has been employed in the theoretical background paper [11]. Furthermore, it combines an initial static loading and a subsequent transient part, and therefore activates potential drift in the actuator force signal and high-frequency vibrations, respectively.

\subsubsection{Wind turbine model}

The stroke amplifying IFF control scheme described in Section 2.3 is used to impose pure viscous damping with an amplified deformation of the viscous damper. In [12] the hybrid viscous damper with the stroke amplifying IFF control is proposed for damping of offshore wind turbines, and the wind turbine model in the Offshore Code Comparison Collaboration (OC3) [18] is therefore also considered in the present paper. The wind turbine illustrated in figure 4 (a) has a tower approximately $78 \mathrm{~m}$ tall, which is positioned on a monopile at $10 \mathrm{~m}$ above Mean Sea Level (MSL) located at $20 \mathrm{~m}$ water depth. The nacelle of the turbine is positioned at the top of the tower and connected to the blades through the hub. The wind turbine is assumed at standstill, whereby the mass of the nacelle, the hub and the blades can be lumped at the top of the tower, and the combined mass is assumed to be 350 ton. The wind turbine structure is modeled by beam elements. Because only planar vibrations in the side-side direction of the turbine are considered the transverse displacement and the associated cross section rotation represent the two nodal degrees of freedom. Thus, the numerical model in the present RTHS study only contains 8 degrees of freedom, as illustrated in figure 4(b). This is significantly less than in the original numerical model of the OC3 reference wind turbine in [12]. However, the dominant dynamics are captured with this model, and the reduced size of the numerical model in the present study is required to limit the computation time during each time step in the RTHS to within a feasible level. The stiffness matrix $\mathbf{K}$ of 
(a)
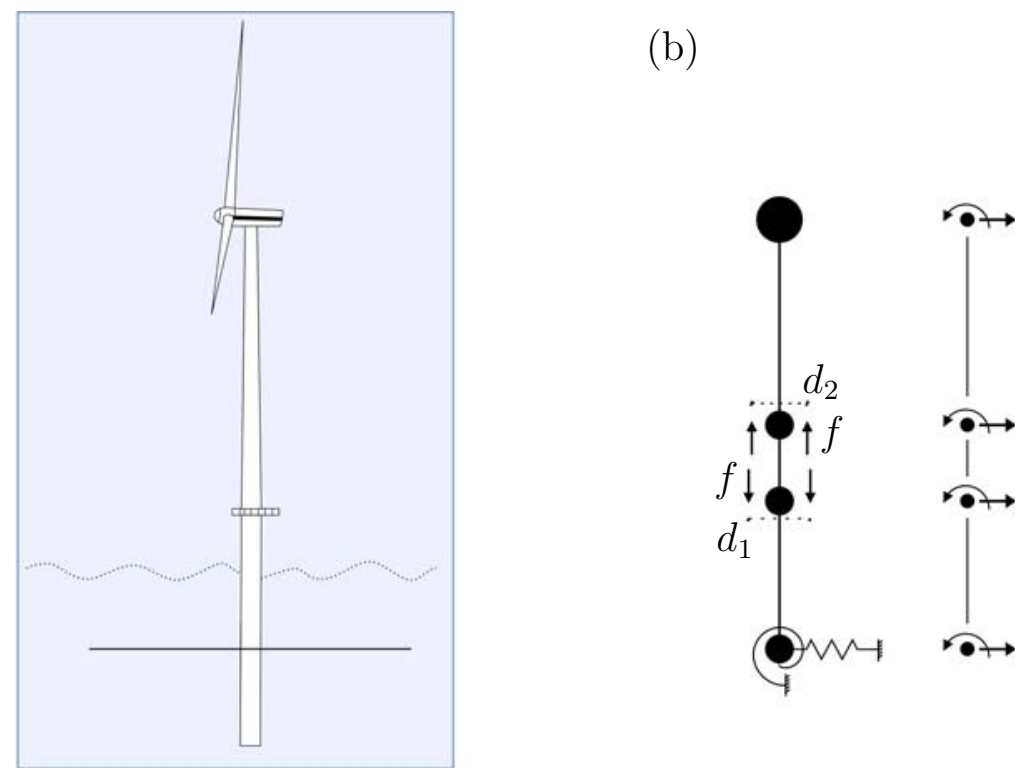

Figure 4: Diagram of (a) the Wind turbine with hybrid damper and (b) corresponding FE model with damper force $f$ and the eight dof shown explicitly.

the wind turbine model is derived from a complementary energy approach, which allows for shear flexibility and varying cross section properties of the wind turbine tower. Furthermore, the flexible foundation of the soil is modeled by a coupled spring foundation model with both lateral and rotational springs at the seabed, which are tuned so that the natural frequency of the first tower mode corresponds to the value used in the reference OC3 study [18]. The mass matrix $\mathbf{M}$ is established by simply lumping the mass of each element and similarly lumping the mass and inertia of the nacelle, rotor and blades at the top node of the tower. Finally, the damper is connected to the tower wall at the bottom of the wind turbine tower and to the tower wall four meters above the bottom of the tower. Hereby, the connectivity vector is given as

$$
\mathbf{w}=\left[0,0,0,-d_{1} / 2,0, d_{2} / 2,0,0\right]^{T}
$$

The non-zero values in $\mathbf{w}$ are located at the rotational degrees of freedom of the two nodes connected by the hybrid viscous damper. 
By inserting the expression for the damper force in (21) and the feedback relation in (14) into the equation of motion in (20) the combined closedloop equation for the structural degrees of freedom $\mathbf{u}(t)$ and the actuator displacement $q(t)$ can be written in a form slightly different from that in (24) for the filtered IFF strategy,

$$
\begin{aligned}
{\left[\begin{array}{ll}
\mathbf{M} & \mathbf{0} \\
\mathbf{0}^{T} & \tau_{q}
\end{array}\right]\left[\begin{array}{c}
\ddot{\mathbf{u}} \\
\ddot{q}
\end{array}\right]+} & {\left[\begin{array}{cc}
c_{n} \mathbf{w} \mathbf{w}^{T} & -c_{n} \mathbf{w} \\
\nu \mathbf{w}^{T} & 1-\nu
\end{array}\right]\left[\begin{array}{c}
\dot{\mathbf{u}} \\
\dot{q}
\end{array}\right] } \\
+ & {\left[\begin{array}{cc}
\mathbf{K} & \mathbf{0} \\
\mathbf{0}^{T} & \omega_{q}
\end{array}\right]\left[\begin{array}{l}
\mathbf{u} \\
q
\end{array}\right]=\left[\begin{array}{c}
\mathbf{f}-\mathbf{w} f_{e}^{m} \\
0
\end{array}\right] }
\end{aligned}
$$

As explained in [12] the optimum viscous damping parameter for a given gain level $\nu$ can be estimated from the expression

$$
c_{o p t}=\frac{2 \Delta \omega_{\infty}}{\gamma^{2}}(1-\nu)
$$

In this expression $\gamma=\sqrt{\left(\mathbf{w}^{T} \mathbf{u}_{1}\right)^{2}}$ represents the modal amplitude across the damper, where $\mathbf{u}_{1}$ is the undamped mode shape vector scaled to unit modal mass. Furthermore, $\Delta \omega_{\infty}=\omega_{1}-\omega_{1, \infty}$ is the difference between the undamped natural frequency $\omega_{1}$ of the side-side mode without damper and the natural frequency $\omega_{1, \infty}$ obtained when the damper acts as a rigid link, see [19]. Finally, the viscous parameters $c_{e}$ and $c_{n}$ are again determined from the interpolation formula (22).

In the present tests the load consist of a wave train of three regular sine waves with wave period $T_{w}=10 \mathrm{~s}$, whereafter the external loading is removed abruptly. The wave train acts at MSL and the load vector is therefore given as

$$
\mathbf{f}(t)=p(t)[0,0,1,0, \ldots]^{T},
$$

where the time dependent load intensity $p(t)$ is now given as

$$
\frac{p(t)}{p_{0}}=\left\{\begin{array}{cc}
\sin \left(2 \pi t / T_{w}\right) & , \quad t \leq 3 T_{w} \\
0 & , \quad t>3 T_{w}
\end{array},\right.
$$

with $p_{0}$ representing the amplitude of the sinusoidal load. 


\subsection{Experimental substructure}

The experimental substructure consist of a physical model of the hybrid viscous damper. As explained in the introduction the idealized viscous dashpot is in the experimental substructure replaced by a semi-active MR damper, which is regulated to emulate the velocity proportional damper force of the ideal viscous dash-pot. The MR damper is placed in series with a hydraulic actuator and a load cell, as shown in figure 5. The load cell measures the damper force $f_{e}^{m}(t)$ produced by the MR damper in the experimental substructure, and the interface between the experimental substructure and the numerical substructure is realized by a hydraulic actuator through a high performance Speedgoat/xPC real-time kernel.

The actuator is equipped with an internal Linear Variable Differential Transformer (LVDT), which measures the actuator displacement and thereby the deformation of the MR damper. The measured actuator displacement is controlled in an inner loop by a SC6000 controller and in an outer loop by a robust integrated actuator control strategy. The inner loop stabilizes the actuator, while the outer loop guarantees that the desired damper deformation $(u-q)$ derived from the numerical substructure and the control equation is implemented appropriately, see [20]. Figure 6 shows the MR damper, which is a RD-8041-1 model produced by LORD company, with a specified peak to peak damper force of approximately $2.4 \mathrm{kN}$ when subjected to a velocity of $0.05 \mathrm{~m} / \mathrm{s}$ at $1 \mathrm{~A}$ current input. To reproduce the performance

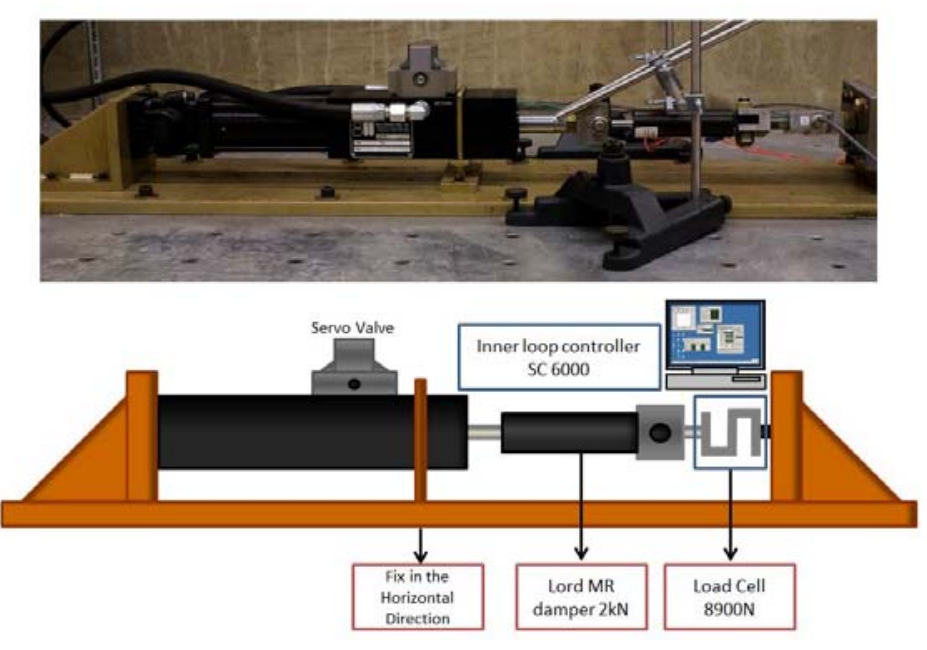

Figure 5: Experimental setup 


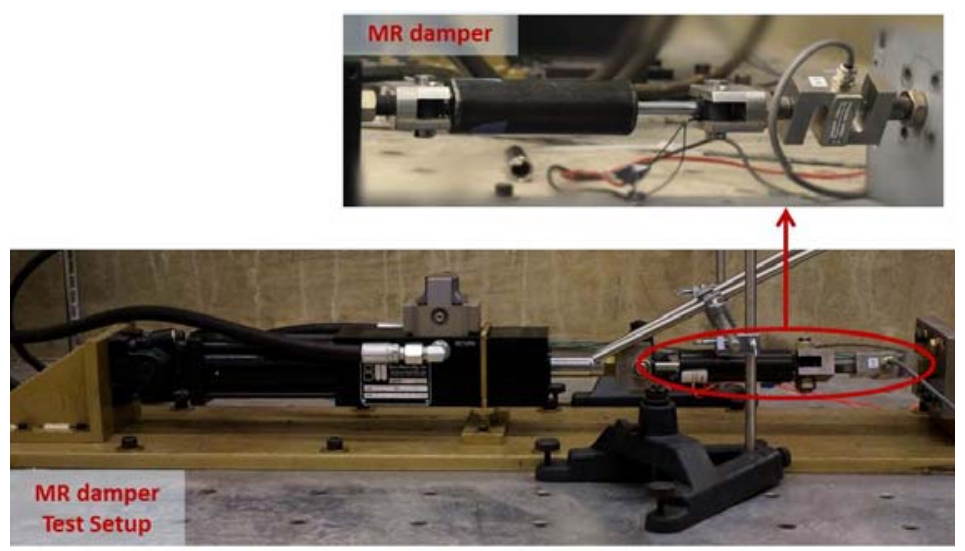

Figure 6: MR damper test setup

of the hybrid viscous damper the MR damper is controlled using a bang-bang feedback control algorithm. This bang-bang control emulates the behavior of the idealized viscous dash-pot by tracking the desired force $f_{e}^{d}$ of the ideal viscous damper contained in the experimental substructure. The bang-bang controller compares the measured damper force $f_{e}^{m}$ from the load cell with the desired force $f_{e}^{d}$ obtained by the control equation, and determines the command current $I$ that allows the actual force in the MR damper $f_{e}^{m}$ to track the corresponding desired force $f_{e}^{d}$. The bang-bang control is an onoff strategy, where maximum current $I=I_{\max }$ is applied when $f_{e}^{m}<f_{e}^{d}$, while $I=0$ in the opposite case when $f_{e}^{m}>f_{e}^{d}$. Furthermore, in order to account for the large variation in the desired damper force $f_{e}^{d}$ the force tracking also involves a nonlinear force mapping from the numerical model of the MR damper applied to the experimental substructure. The force measured in the experimental setup is multiplied with an appropriate factor before it is sent to the numerical substructure, and the computed displacement from the numerical substructure is subsequently divided by the same factor before it is sent to the actuator in the experimental setup. Further details on this nonlinear mapping can be found in the accompanying paper [21].

A schematic overview of the RTHS setup is provided in the block diagram in figure 7. The numerical domain is constituted by the Finite Element model in the block diagram. It represents the governing equations of motion, i.e. (24) for the shear frame structure with the filtered IFF control of the hybrid viscous damper and (27) for the wind turbine model with the pure IFF and stroke amplification. The input to this numerical model is the external load 
f and the measured damper force $f_{e}^{m}$ from the load cell. The output of this block is the computed displacements and velocities, and in particular the deformation of the hybrid viscous damper $u=\mathbf{w}^{T} \mathbf{u}$ and its velocity component $\dot{u}=\mathbf{w}^{T} \dot{\mathbf{u}}$. The desired motion of the actuator in the hybrid viscous damper is determined by the Force feedback controller block, which receives the measured damper force $f_{e}^{m}$ as input. This block therefore represents the real-time control and is therefore considered as part of the experimental substructure, although the actuator motion is calculated numerically according to the control laws. The output of this block is the desired actuator displacement $q_{d}$ and its velocity $\dot{q}_{d}$ (with subscript $d$ for desired), determined by solving (10) for the shear frame with filtered IFF control and (14) for the wind turbine with the stroke amplifying IFF. Note that in both (10) and (14) the input is only the experimentally measured damper force $f_{e}^{m}$, due to the partitioning in $(21)$.

In the RTHS setup the hydraulic actuator at the interface must provide the correct displacement $u-q_{d}$ across the MR-damper, while the bang-bang control determines the MR-damper current $I$ to simultaneously secure the desired viscous damper force. The MR-damper deformation $u-q_{d}$ is therefore passed on to the hydraulic actuator, which simultaneously acts as the active actuator in the hybrid viscous damper with command stroke $q_{d}$ and the dedicated interface actuator, transferring the displacement $u$ from the numerical substructure to the experimental substructure. The resulting displacement of the hydraulic actuator is therefore given as the sum of the command displacement $-q_{d}$ from the force feedback controller and the hybrid damper displacement $u=\mathbf{w}^{T} \mathbf{u}$ computed by the Finite Element model in the numerical substructure. The desired viscous damper force $f_{e}^{d}=c_{e}\left(\dot{u}-\dot{q}_{d}\right)$ is computed and used as input to the Bang-bang controller, which controls the MR damper via the damper current $I$ to emulate $f_{e}^{d}$. The output from the hybrid damper model is the damper force $f_{e}^{m}$ measured by the load cell in the experimental substructure.

\section{Results of Real Time Hybrid Simulations}

This section presents results from the Real time hybrid simulations (rths) with the hybrid viscous damper concept using the experimental setup with the bang-bang control of the MR damper described in Section 3.2. The experimental results are compared to the results from the equivalent numerical simulations associated with the interpolation parameter $\eta=0$. Initially, the 


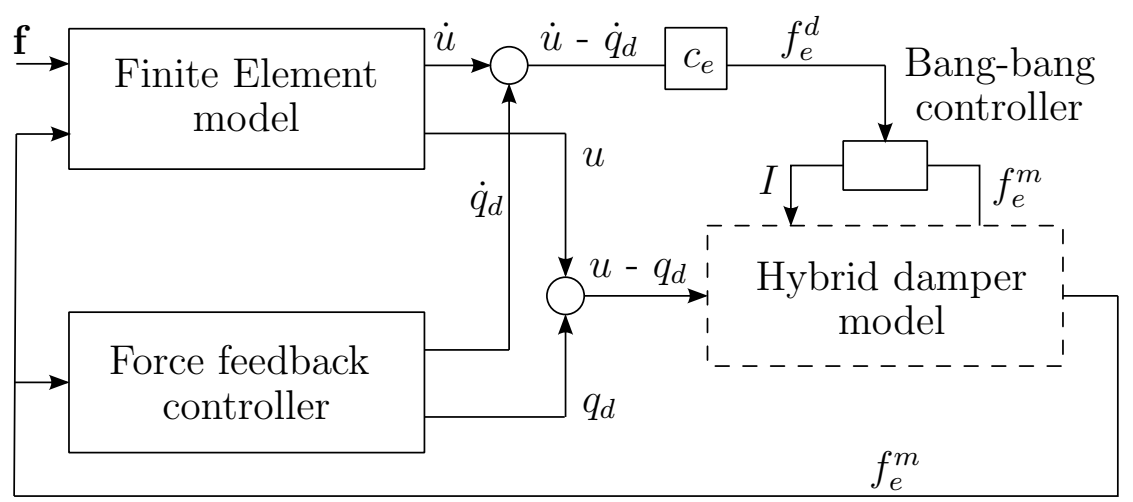

Figure 7: Schematic overview of the RTHS setup in block diagrams

performance of the hybrid damper with the filtered IFF controller is considered, and subsequently the performance of the pure IFF controller for damper stroke amplification is assessed. Finally, the performance of the bang-bang controller used to track the desired viscous damper force is discussed separately. It should be mentioned that the specific performance of the SC6000 controller is not evaluated, as it is considered as a standard component of the corresponding actuator, while the performance of the Speedgoat/xPC kernel is discussed in the accompanying paper [21].

\subsection{Hybrid damper with filtered IFF controller}

As explained in Section 3.1.2 the analysis of the filtered IFF controller is conducted for a hybrid viscous damper positioned between the first and second floor in a shear frame structure, which is loaded by the ramp load described in (26). To be able to asses both the performance of the hybrid viscous damper and the influence of the corner frequency $\omega_{q}$ in $(10)$ the hybrid simulations are performed with gain values of $\nu=-2.0$ and $\nu=-4.0$, and with corner frequencies $\omega_{q}=\omega_{1} / 20, \omega_{1} / 8$ and $\omega_{1} / 5$. Furthermore, a simulation with $\nu=0$ has been conducted in order to specifically address the performance of the bang-bang controller without any influence of the filtered IFF controller. For all RTHS's an interpolation parameter of $\eta=0.5$ in (22) has been used.

\subsubsection{Actuator drift and corner frequency}

Initially, the performance of the filtered integration in (10) and the corresponding corner frequency $\omega_{q}$ is emphasized. Figure 8 compares both the 
(a)

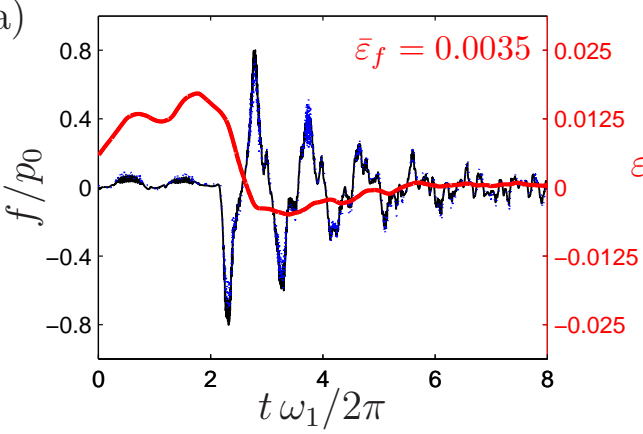

(c)

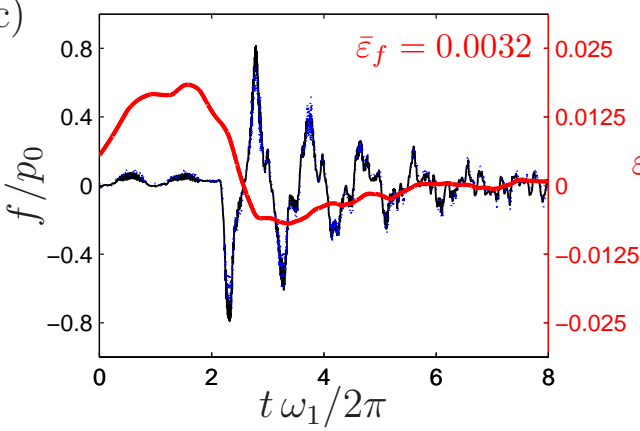

(e)

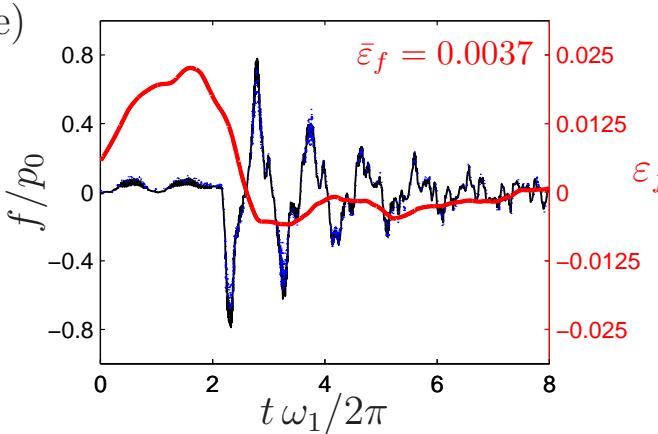

(b)

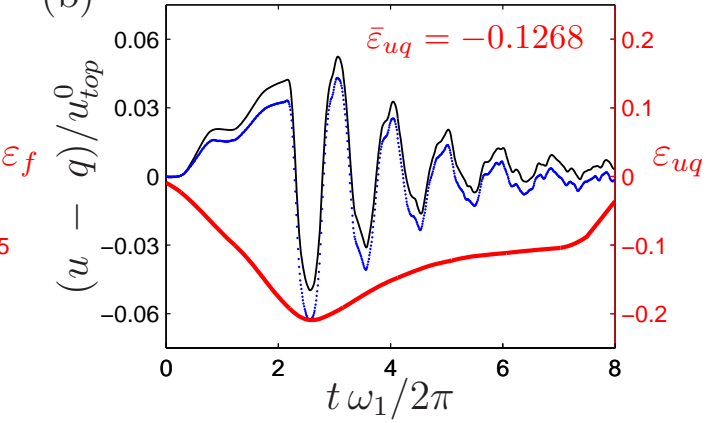

(d)

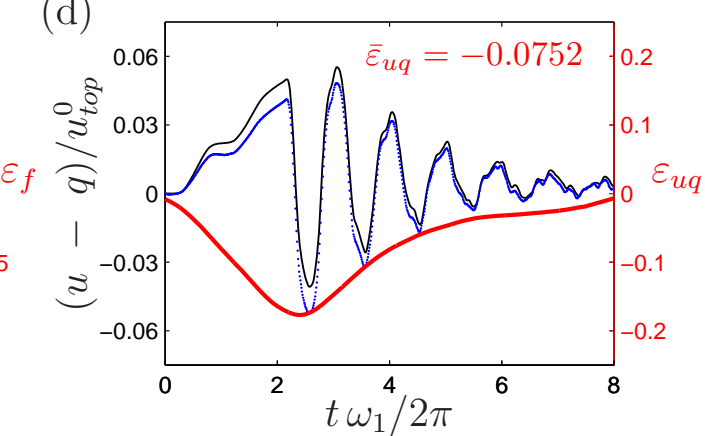

(f)

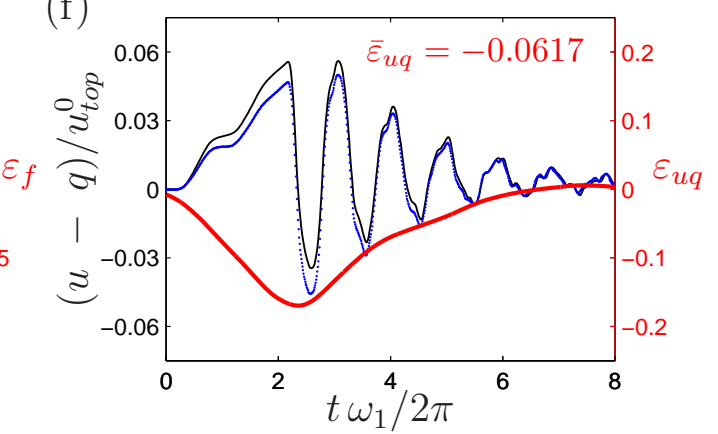

Figure 8: Measured damper force $f_{e}^{m}(\cdot \cdot)$ compared with desired damper force $f_{e}^{d}(-)$ and desired damper displacement $u-q_{d}(\cdot \cdot)$ compared with numerical damper displacement ( - ) for filtered IFF controller with $\nu=-2.0$ and $\omega_{q}=\omega_{1} / 20(\mathrm{a}, \mathrm{b}), \omega_{1} / 8(\mathrm{c}, \mathrm{d})$ and $\omega_{1} / 5(\mathrm{e}, \mathrm{f})$, respectively. Error measure for damper force $\varepsilon_{f}$ and damper displacement $\varepsilon_{u q}$ given by (-). 
desired damper force $f_{e}^{d}$ (black solid curves) with the measured damper force $f_{e}^{m}$ (blue dots) produced by the MR damper (a,c,e) and the desired damper displacement $u-q_{d}$ (blue dots) sent to the experimental substructure with the corresponding displacement $u-q$ (black solid curves) obtained by a direct time integration of the equations of motion in (24) with $\eta=0$, and thereby $f_{e}^{m}=0(\mathrm{~b}, \mathrm{~d}, \mathrm{f})$. The difference between the desired and measured damper force represents the error in the force tracking, while the difference between the damper displacement from RTHS and that from numerical simulations represents the drift of the actuator displacement. The primary goal of this section is to investigate how an error in the force tracking influences the drift in the damper displacement. The results are presented for $\nu=-2.0$ and with corner frequencies $\omega_{q}=\omega_{1} / 20(\mathrm{a}, \mathrm{b}), \omega_{1} / 8(\mathrm{c}, \mathrm{d})$ and $\omega_{1} / 5(\mathrm{e}, \mathrm{f})$.

It follows from figure $8(\mathrm{a}, \mathrm{c}, \mathrm{e})$ that the force tracking is in fact quite accurate, as the measured force $f_{e}^{m}$ (blue dots) in general seems to track the desired force $f_{e}^{d}$ (black solid curves) quite well. However, the force tracking curves show some fluctuations around the local peaks because the bang-bang controller tends to slightly overshoot the desired damper force. Furthermore, a deviation is also observed in figure $8(\mathrm{~b}, \mathrm{~d}, \mathrm{f})$ between the desired displacement over the MR damper $u-q_{d}$ (blue dots) and the numerically determined displacement $u-q$ over the equivalent viscous damper (black solid curves). However, this error appears to be quite smooth because of the filtered integration in the feedback relation (10), which acts as a low pass filter.

The red solid curves in figure 8 represent the relative error in the force tracking $\varepsilon_{f}=\left(f_{e}^{m}-f_{e}^{d}\right) / f_{e, \max }^{d}(\mathrm{a}, \mathrm{c}, \mathrm{e})$ and the corresponding error in the damper displacement $\varepsilon_{u q}=\left(u-q_{d}-(u-q)\right) /(u-q)_{\max }(\mathrm{b}, \mathrm{d}, \mathrm{e})$, with the scaling indicated on the right abscissae. To specifically show the low frequency content, leading to the drift in actuator displacement, both error signals $\varepsilon_{f}$ and $\varepsilon_{u q}$ have been filtered twice by the Matlab function conv with a rectangular window function of length $T_{1}=2 \pi / \omega_{1}$, corresponding to a single vibration period of the first mode shape. Furthermore, to quantify the errors the mean values of both the force error $\bar{\varepsilon}_{f}$ and the displacement error $\bar{\varepsilon}_{u q}$ are given in figure 8 for each simulation.

When comparing the two error signals during the first two vibration periods where the load is ramped $\left(t<4 \pi / \omega_{1}\right)$ there appears to be a tendency that a positive force error $\varepsilon_{f}>0$ coincides with a negative slope in the displacement error $d \varepsilon_{u q} / d t<0$. After the two initial periods the external load is removed and the error in the damper force in general becomes smaller $\left(\varepsilon_{f} \rightarrow 0\right)$, which agrees with the magnitude of the displacement error also 
becoming smaller $\left(\varepsilon_{u q} \rightarrow 0\right)$. Furthermore, it is observed that for increasing values of the corner frequency $\omega_{q}=\omega_{1} / 20(\mathrm{~b})<\omega_{1} / 8(\mathrm{~d})<\omega_{1} / 5(\mathrm{f})$, the magnitude of the displacement error $\varepsilon_{u q}$ descends to zero at a faster rate, whereby the accumulated drift in the damper displacement is reduced. Finally, because the three curves for the force error $\varepsilon_{f}$ in figure 8(a,c,e) seems to be also identical, the observed reduction in drift of the damper displacement in figure $8(\mathrm{~b}, \mathrm{~d}, \mathrm{f})$ is most likely due to the increase in corner frequency $\omega_{q}$. Thus, it is important to choose $\omega_{q}$ sufficiently large to avoid damper drift without deteriorating the performance of the hybrid damper concept.

Figure 9 shows the same results as in figure 8, but now for $\nu=-4.0$. Again there is a tendency that a positive force error $\varepsilon_{f}$ coincides with a negative slope in $\varepsilon_{u q}$ and vice versa. Thus, it can again be argued that the error $\varepsilon_{u q}$ is seemingly proportional to the accumulated error in the damper force $\varepsilon_{f}$. However, the three curves for $\varepsilon_{f}$ in figure 9 are in the present case not similar, which means that the corresponding magnitudes of the displacement errors $\varepsilon_{u q}$ are difficult to compare. Instead the individual mean values in the force signal $\bar{\varepsilon}_{f}$ may be compared to the associated mean values $\bar{\varepsilon}_{u q}$ of the displacement errors. For $\omega_{q}=\omega_{1} / 20$ the error ratio is $\left|\bar{\varepsilon}_{u q} / \bar{\varepsilon}_{f}\right|=|0.0424 / 0.0004|=$ 106 , while for $\omega_{q}=\omega_{1} / 8$ it reduces to $\left|\bar{\varepsilon}_{u q} / \bar{\varepsilon}_{f}\right|=|0.0254 / 0.0005|=51$, while it for the largest corner frequency $\omega_{q}=\omega_{1} / 5$ the ratio is further reduced to $\left|\bar{\varepsilon}_{u q} / \bar{\varepsilon}_{f}\right|=|0.1375 / 0.0058|=23$. Thus, the comparison of the mean error ratios indicates that the drift in the actuator signal is in fact reduced for increasing values of the corner frequency $\omega_{q}$.

\subsubsection{Damping performance}

Figure 10 shows the displacement response $u_{t o p}$ of the shear frame top floor. As for the damper displacement results in figures 8 and 9 the response computed by the RTHS is in the figure compared to the response obtained by a direct time integration of the equations of motion (24) with $\eta=0$. The three pair of curves in figure 10 represent the three non-dimensional gain values $\nu=0.0,-2.0$ and -4.0 , while the corner frequency is $\omega_{q}=\omega_{1} / 8$ for all curves in figure 10.

Although there are small discrepancies between the hybrid simulation results and the full numerical results, the agreement between the results is in general very good. As illustrated by the dashed exponential decaying curves the experimental results verify that for $\nu<0$ the apparent damping increases when the magnitude of the negative non-dimensional gain increases $\nu \rightarrow-\infty$. The damping ratio is estimated by the logarithmic decrement 
(a)

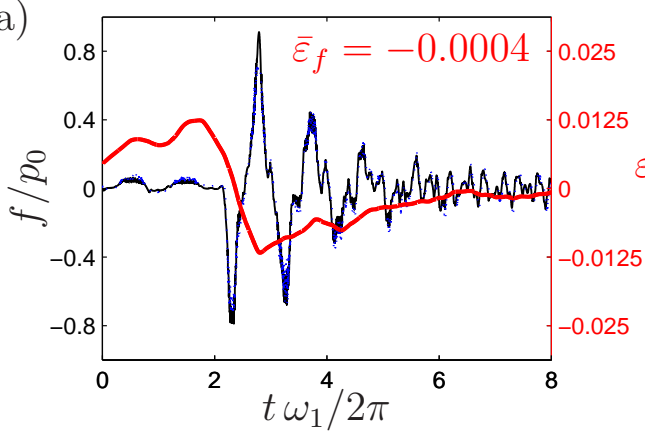

(c)

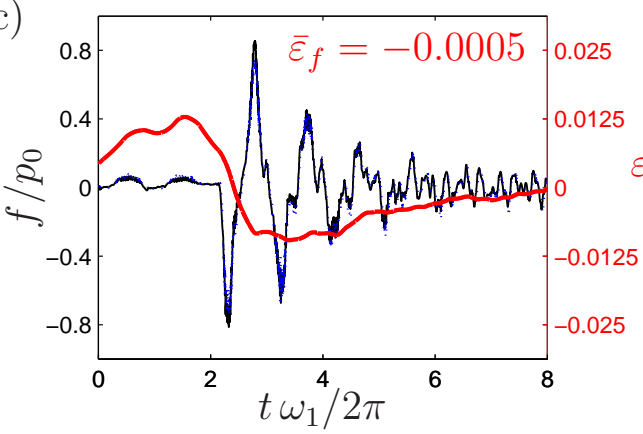

(e)

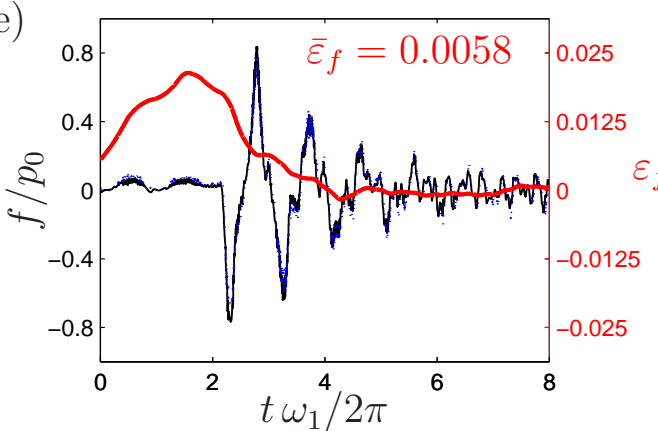

(b)

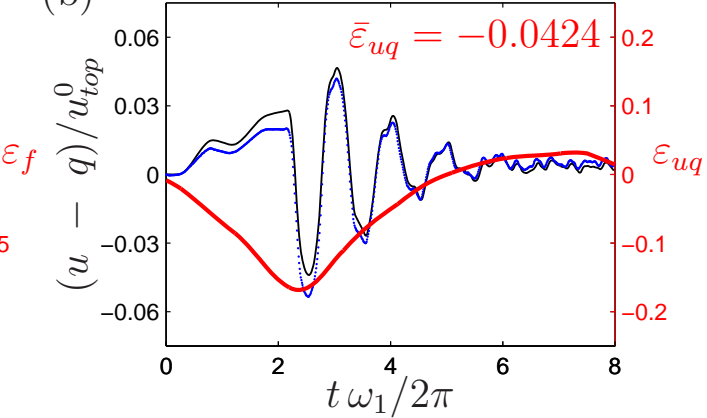

(d)

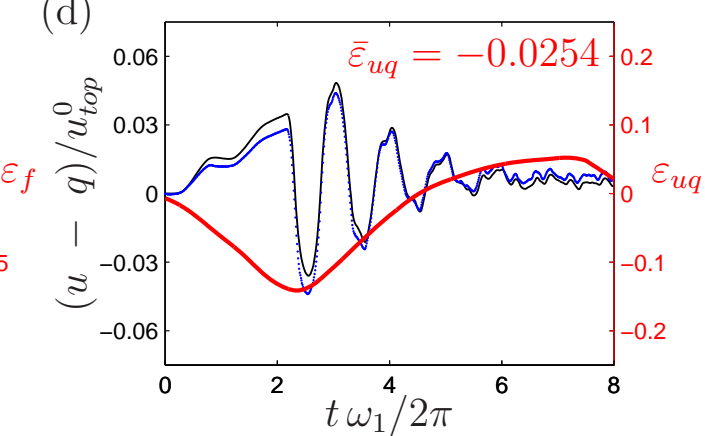

(f)

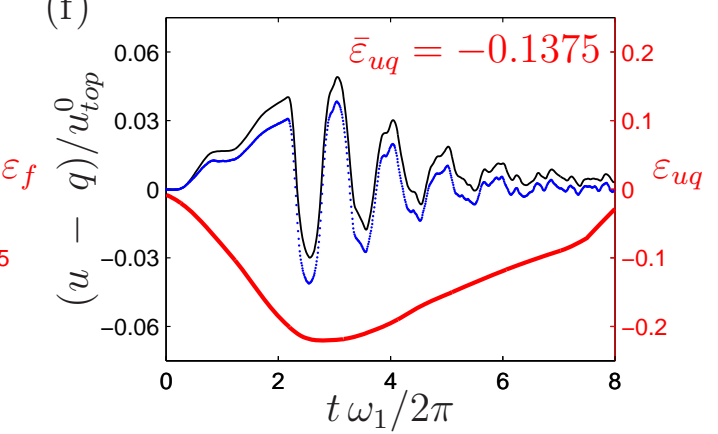

Figure 9: Measured damper force $f_{e}^{m}(\cdot \cdot)$ compared with desired damper force $f_{e}^{d}(-)$ and desired damper displacement $u-q_{d}(\cdot \cdot)$ compared with numerical damper displacement (-) for filtered IFF controller with $\nu=-4.0$ and $\omega_{q}=\omega_{1} / 20(\mathrm{a}, \mathrm{b}), \omega_{1} / 8(\mathrm{c}, \mathrm{d})$ and $\omega_{1} / 5(\mathrm{e}, \mathrm{f})$, respectively. Error measure for damper force $\varepsilon_{f}$ and damper displacement $\varepsilon_{u q}$ given by (-). 
evaluated between both the first and fourth maximum and minimum in the free vibration response. For the direct time integration this procedure gives the average damping ratios $\zeta=0.053,0.086$ and 0.108 for $\nu=0.0,-2.0$ and -4.0 , respectively. The corresponding damping ratios obtained in [11] by direct time integration with $\omega_{q}=0$ are $\zeta=0.053,0.093$ and 0.124 . This shows that the presence of the corner frequency $\omega_{q}=\omega_{1} / 8$ slightly reduces the damping performance of the hybrid viscous damper. Specifically, the reduction in damping ratio due to the presence of the corner frequency in the filter equation is $8 \%$ for $\nu=-2.0$, while it is $13 \%$ for the larger gain $\nu=-4.0$. This shows that the deterioration in damping performance due to a non-vanishing $\omega_{q}$ becomes more pronounced for increasing magnitude of negative $\nu$.

For the response obtained by the RTHS the damping ratios are estimated by the logarithmic decrement procedure to be $\zeta=0.051,0.082$ and 0.106 , which are slightly smaller than the damping ratios obtained by the direct time integration. Specifically, the reduction in damping ratio compared to the results obtained by the pure time integration is approximately $4 \%$ for $\nu=0.0$, $5 \%$ for $\nu=-2.0$ and $2 \%$ for the largest negative gain value $\nu=-4.0$. Thus, the deviations between the curves in figure 10, representing the RTHS and the direct time integration, are apparently independent of the magnitude of the gain value $\nu$ and may instead be attributed to the performance of the bang-bang controller. The rather small deviations between the results obtained by the direct time integration and by the hybrid simulation show that the performance of the hybrid viscous damper is quite robust with respect to
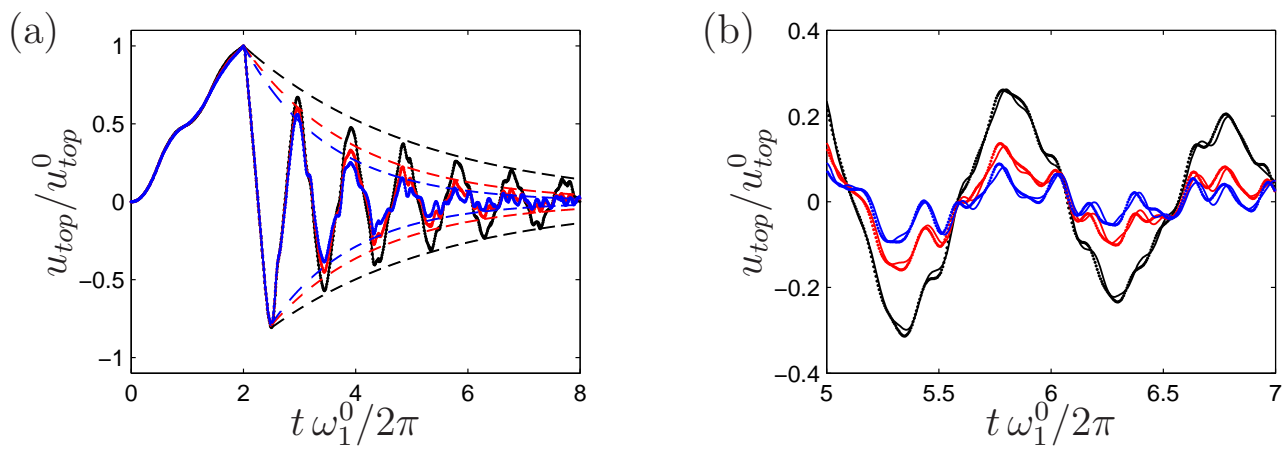

Figure 10: Top-floor response by numerical simulation for $\nu=0.0(-),-2$ $(-)$ and $-4(-)$ and by RTHS for $\nu=0.0(\cdot \cdot),-2(\cdot)$ ) and $-4(\cdot)$ ) and with $\omega_{q}=\omega_{1} / 8$ 
errors in the force tracking. However, It should be noted that the interpolation factor $\eta=0.5$, whereby only half of the total damper force is extracted from the experimental substructure.

\subsection{Hybrid damper with stroke amplifying IFF controller}

This section investigates the hybrid viscous damper with stroke amplifying IFF control, as presented in Section 2.3. The hybrid damper is installed at the bottom of the tower of an offshore wind turbine model and loaded by three sinusoidal waves, as explained in Section 3.1.3. The control gain values in the RTHS are chosen as $\nu=0.5$ or $\nu=0.75$, while the filter frequencies of the control equation in (14) are either $\omega_{f}=\tau_{f} \omega_{1}^{2}=\omega_{1} / 8$ or $\omega_{1} / 5$. These parameter combinations assess both the performance of the hybrid viscous damper and the filtered integration with $\omega_{f}=\tau_{f} \omega_{1}^{2}>0$. A small interpolation parameter of $\eta=0.1$ has been used in all the hybrid simulations associated with the stroke amplifying IFF control.

\subsubsection{Actuator drift and filter parameters}

The performance of the filtered version of the pure IFF control in (14) is investigated by comparing the measured damper force $f_{e}^{m}$ of the MR damper with the desired damper force $f_{e}^{d}$, and furthermore by comparing the desired damper displacement $\left(u-q_{d}\right)$ sent to the experimental substructure with the corresponding displacement $(u-q)$ obtained by direct time integration of (27) with $\eta=0$. The results of the simulations are presented for $\nu=0.5$ in figure 11 and for $\nu=0.75$ in figure 12. The curves in figure 11(a,b) are obtained with filter frequency $\omega_{f}=\tau_{f} \omega_{1}^{2}=\omega_{1} / 8$, while in $(\mathrm{c}, \mathrm{d})$ the frequency is $\omega_{f}=\tau_{f} \omega_{1}^{2}=\omega_{1} / 5$. The small deviation between the desired and measured damper force, which mainly occur around the maxima and minima, is again attributed to the limited force tracking capabilities of the bang-bang control. The associated difference in damper displacement is seen to be somewhat larger than for the force tracking error, but with less irregularities. The latter can be ascribed to the integration in (14), which acts as a low pass filter. The error in damper displacement represents the actuator drift in the hybrid viscous damper, which may cause actuator saturation if it becomes too large. When comparing the desired damper displacement $\left(u-q_{d}\right)$ for $\nu=0.5$ in figure 11 and for $\nu=0.75$ in figure 12 it is found that the damper amplitude is approximately doubled, which agrees with the theoretical result in (19). 
The red solid curves in figures 11 and 12 show the relative force tracking error $\varepsilon_{f}=\left(f_{e}^{m}-f_{e}^{d}\right) / f_{e, \max }^{d}$ and the relative error in the damper displacement $\varepsilon_{u q}=\left(\left(u-q_{d}\right)-(u-q)\right) /(u-q) \max$, with the scaling shown on the right abscissae. The comparison of the mean force tracking errors $\bar{\varepsilon}_{f}$ and $\bar{\varepsilon}_{u q}$ gives an indication of the influence of the choice of filter frequency $\omega_{f}$. For $\nu=0.5$ the error ratios are $\bar{\varepsilon}_{u q} / \bar{\varepsilon}_{f} \simeq 5$ and 2.7 for $\omega_{f}=\tau_{f} \omega_{1}^{2}=\omega_{1} / 8$ and $\omega_{1} / 5$, respectively, while for $\nu=0.75$ they are $\bar{\varepsilon}_{u q} / \bar{\varepsilon}_{f} \simeq 6.3$ and 3.9. The apparent inverse proportionality between the error ratio and the corresponding magnitude of the filter frequency $\omega_{f}$ indicates that the drift can be effectively reduced by increasing the value of $\omega_{f}=\tau_{f} \omega_{1}^{2}$, while the drift of the actuator seems to increase with the gain parameter $\nu$.
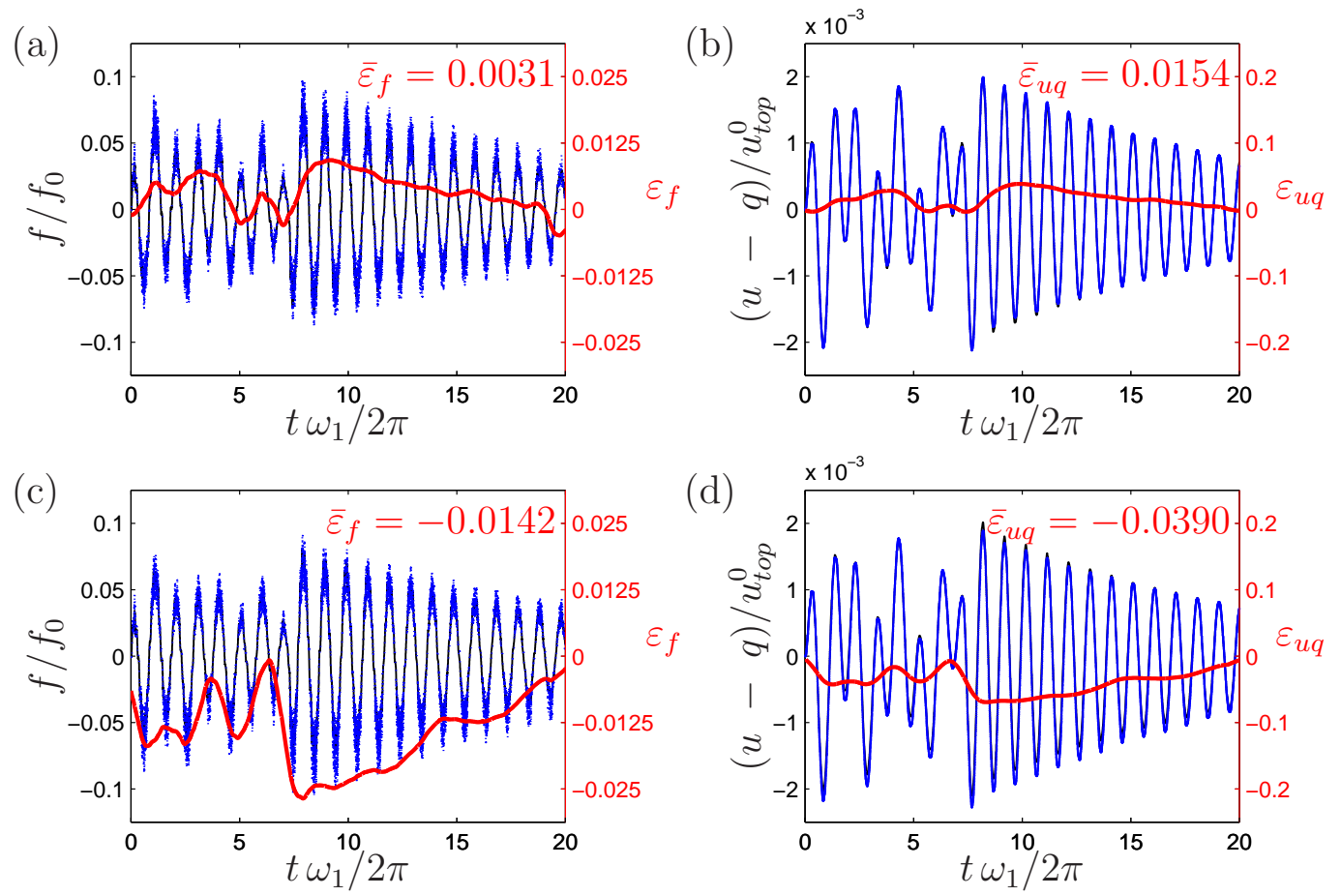

Figure 11: Measured damper force $f_{e}^{m}(\cdot \cdot)$ compared with desired damper force $f_{e}^{d}(-)$ and desired damper displacement $u-q_{d}(\cdot \cdot)$ compared with numerical damper displacement (-) for $\nu=0.5$ and $\omega_{f}=\tau_{f} \omega_{1}^{2}=\omega_{1} / 8$ (a)-(b) and $\omega_{1} / 5$ (c)-(d). Error measure for damper force $\varepsilon_{f}$ and damper displacement $\varepsilon_{u q}$ given by (-). 

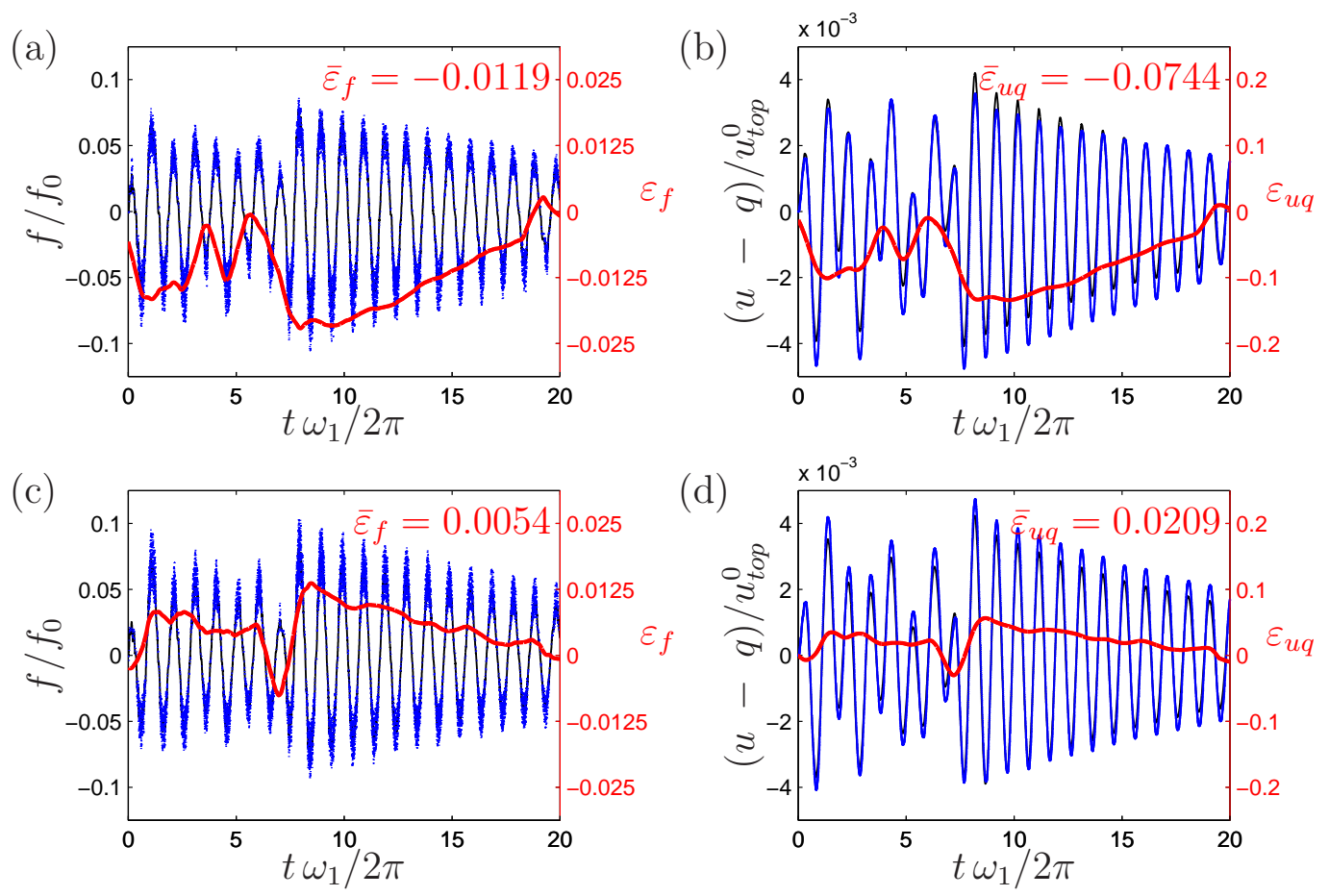

Figure 12: Measured damper force $f_{e}^{m}(\cdot)$ compared with desired damper force $f_{e}^{d}(-)$ and desired damper displacement $u-q_{d}(\cdot \cdot)$ compared with numerical damper displacement (-) for $\nu=0.75$ and $\omega_{f}=\tau_{f} \omega_{1}^{2}=\omega_{1} / 8$ (a)-(b) and $\omega_{1} / 5$ (c)-(d). Error measure for damper force $\varepsilon_{f}$ and damper displacement $\varepsilon_{u q}$ given by (-).

\subsubsection{Damping performance}

The damping performance of the hybrid damper with the IFF controller is investigated by comparing the hybrid simulation results $(\cdot \cdot)$ of the tower top displacement with the corresponding numerical results (-). The results are shown in figure 13. The free decay is estimated from the exponentially decaying curves in the figure, which are seen to almost exactly match the corresponding decay in vibration amplitude. The results in the figure are shown for gain values (a) $\nu=0.5$ and (b) $\nu=0.75$, while the corner frequency of the integration filter in (14) is chosen as $\omega_{f}=\tau_{f} \omega_{1}^{2}=\omega_{1} / 5$. The experimental and numerical results are seen to match very well, and the damping performance appears to be almost identical for the RTHS and the corresponding numerical simulation. The damping ratio is again estimated by 

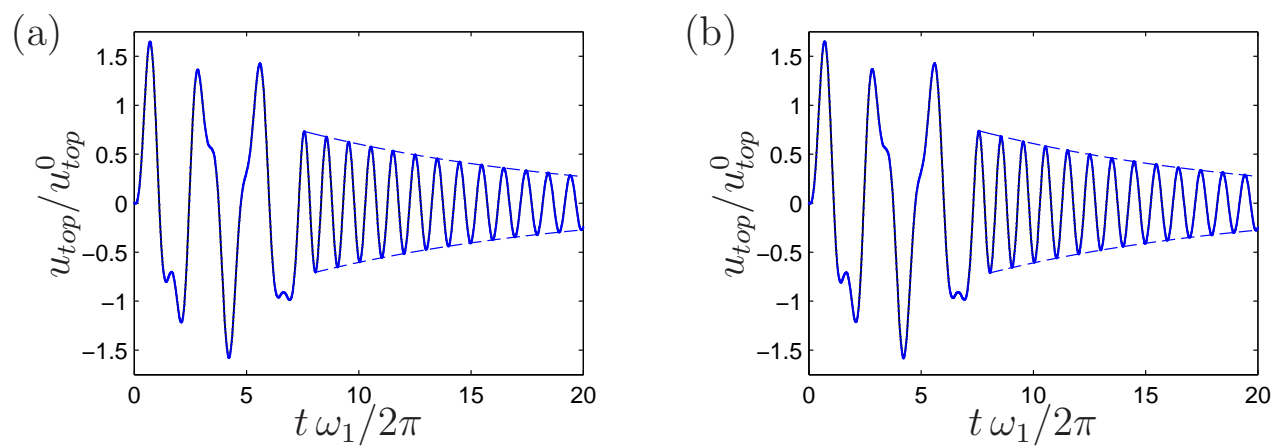

Figure 13: Top tower displacement as computed in the numerical substructure $(\cdot)$, and as computed by a numerical simulation (-) for (a) $\nu=0.5$ and (b) $\nu=0.75$ for $\omega_{f}=\tau_{f} \omega_{1}^{2}=\omega_{1} / 5$

the logarithmic decrement evaluated between the first and fourth maximum and minimum of the free decay. The average damping ratio is $\zeta=0.0128$ for the numerical simulation, while $\zeta=0.0127$ is obtained from the RTHS results. Thus, the deviation between the two results is less than $1 \%$. The small reduction in damping ratio observed in the RTHS may as before be due to the bang-bang controller and the non-viscous behavior of the MR damper. Since a fairly small interpolation parameter $\eta=0.1$ has been used in the present study, the performance of the RTHS may be influenced by the larger experimental substructure in the case of a full scale test with different partitioning in the RTHS.

\subsection{Force tracking}

As the performance of the hybrid damper in the experimental substructure is found to depend on the performance of the associated Bang-Bang control of the MR damper a specific analysis of the force tracking capabilities are now conducted. The performance of the Bang-Bang controller is illustrated in figure 14 and figure 15 for the hybrid damper with filtered IFF controller and for the hybrid damper with stroke amplifying IFF controller. The plots show force-velocity response of the MR damper $(\cdot \cdot)$ together with the corresponding desired force-velocity response $(--)$. The results are from the same simulations as the results presented in figure 8 and 9 , and in figure 11 and 12, respectively. In general, the MR damper together with the bangbang control is able to track the desired force response quit well. However, due to the abrupt on/off nature of the bang-bang controller the damper force 

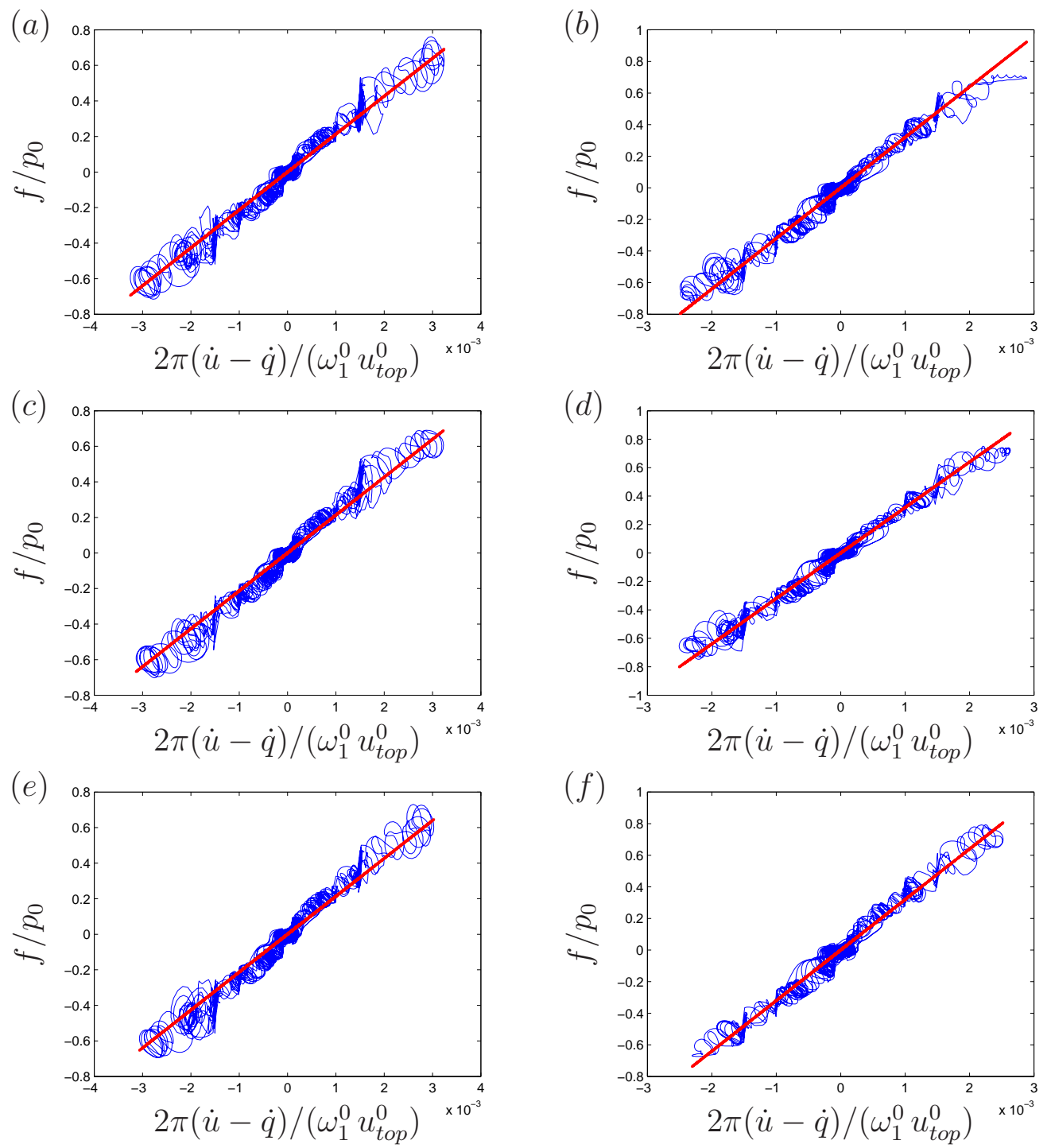

Figure 14: Damper force versus velocity response for the MR damper in the experimental substructure (-) together with the desired response $(-)$ for the filtered IFF controller. For $\nu=-2$ and $\omega_{q}=\omega_{1} / 20$ (a), $\omega_{1} / 8$ (c) and $\omega_{1} / 5$ (e), and for $\nu=-4$ and $\omega_{q}=\omega_{1} / 20$ (b), $\omega_{1} / 8$ (d) and $\omega_{1} / 5$ (f).

response inherently introduces a certain amount of high-frequency fluctuations. The IFF controller in figure 15 results in a very jagged response, while the filtered IFF controller in figure 14 produces a less noisy force-velocity re- 

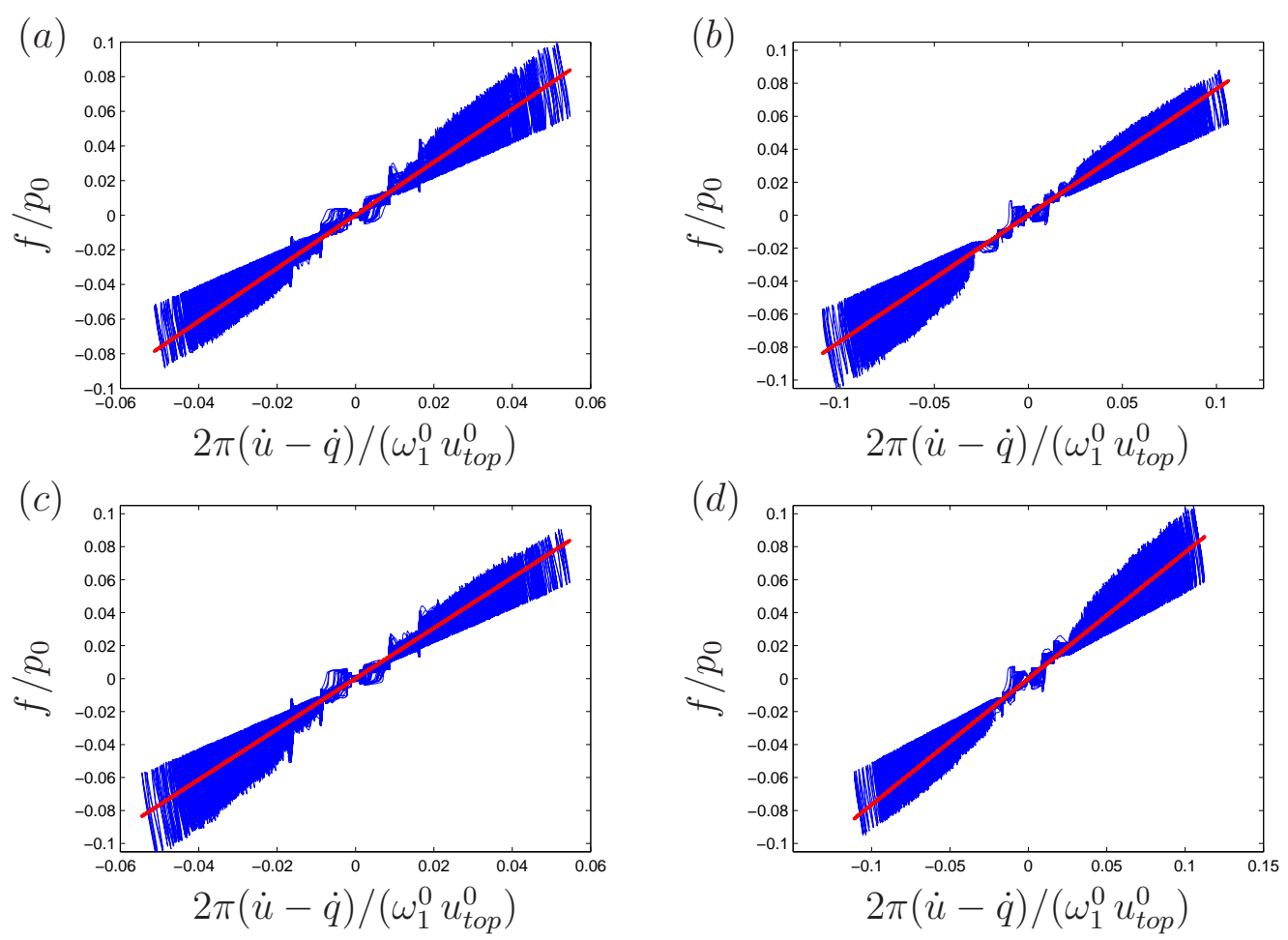

Figure 15: Damper force versus velocity response for the MR damper in the experimental substructure (-) together with the desired response (-) for the IFF controller. For $\nu=0.5$ and $\omega_{f}=\tau_{f} \omega_{1}^{2}=\omega_{1} / 8$ (a) and $\omega_{1} / 5$ (c), and for $\nu=0.75$ and $\omega_{f}=\tau_{f} \omega_{1}^{2}=\omega_{1} / 8(\mathrm{~b})$ and $\omega_{1} / 5(\mathrm{~d})$.

lation. This difference could be due to the controller as well as the difference in the numerical model and loading. As shown in figures 8-9 and 11-12 the force tracking error also contains a low-frequency component, which leads to drift in the actuator displacement. The low-frequency error is caused by a force offset in the MR damper force, most likely due to clamping of the damper. The low-frequency error is amplified by the IFF controller, introducing drift of the actuator displacement, which may result in saturation of the actuator command signal. The non-zero force offset could also be an issue in a real-world implementation of the hybrid damper concept. As also seen in figures 8-9 and 11-12 this undesirable drift is in fact reduced effectively by introduction of a filtered integration with suitably chosen corner frequency $\omega_{q}$ or filter parameters $\omega_{f}$ and $\tau_{f}$, respectively. 


\section{Discussion and outlook}

Overall, the results from the RTHS and the results from the numerical simulations seem to match fairly well. The results from the hybrid simulations with the filtered IFF controller verify that for a negative gain $\nu<0$, damping can be increased compared to the passive viscous case, while the results from the RTHS with the pure IFF controller demonstrate that for $0<\nu<1$ the displacement over the damper is increased. These results validate the numerical results presented in [11] and [12], and show that the hybrid viscous damper concept can be used for damping of flexible structures. Discrepancies between the experimental and numerical results are most pronounced in the results for the displacement over the damper, which are seen to be caused by an error in the damper force tracking capabilities. This tracking error has both a high-frequency part due to the fluctuations in the Bang-Bang controller, and a low-frequency part caused by the force offset in the MR damper. The high-frequency part causes deterioration in the damping efficiency of the hybrid damper. For the hybrid damper with filtered IFF control and $\eta=0.5$ the reduction in damping ratio is approximately $4 \%$, while for the hybrid damper with pure IFF control and interpolation parameter $\eta=0.1$ the decrease is found to be around $1 \%$.

The present paper provides an initial experimental verification of the realization and performance of the hybrid viscous damper with filtered force feedback. Future work on the analysis of the hybrid viscous damper should focus on increasing the damper force ratio in the experimental substructure of the RTHS to make the simulations more realistic. For the present hybrid damper model the MR damper dictates the stability limit. Alternatively, the stability of the RTHS could be improved by simply introducing structural damping to the numerical model represented by the equation of motion in (20). This might also raise the interpolation parameter $\eta$, in particular for the stroke amplifying IFF controller, for which the MR damper response in figure 15 is rather noisy. Furthermore, applying a more advanced controller for the MR damper would also reduce the force tracking error and thereby potentially increase the overall damping performance as well as raise the allowable interpolation parameter $\eta$. Finally, the low-frequency part of the tracking error leads to drift of the actuator command signal. This drift could potentially be decreased by applying an improved controller of the MR damper that does not exhibit such a severe offset in the damper force. 


\section{Acknowledgments}

This work has been supported by the Danish Energy Agency and Vestas Wind Systems A/S under the EUDP project 'Monopile cost reduction and demonstration by joint applied research', and the stay abroad at Purdue University has been supported by 'The Augustinus Foundation' and 'The Oticon Foundation'. Purdue University co-authors where supported in part by the National Science Foundation grant CCF-1136075. The support is greatly appreciated.

[1] B. F. Spencer Jr, S. Nagarajaiah, State of the art of structural control, Journal of structural engineering 129 (7) (2003) 845-856.

[2] T. E. Saeed, G. Nikolakopoulos, J.-E. Jonasson, H. Hedlund, A stateof-the-art review of structural control systems, Journal of Vibration and Control.

[3] G. W. Housner, L. A. Bergman, T. K. Caughey, A. G. Chassiakos, R. O. Claus, S. F. Masri, R. E. Skelton, T. T. Soong, B. F. Spencer Jr., J. T. P. Yao, Structural control: Past, present, and future, Journal of Engineering Mechanics 123 (9) (1997) 897-971.

[4] M. S. Miah, E. N. Chatzi, F. Weber, Semi-active control for vibration mitigation of structural systems incorporating uncertainties, Smart Materials and Structures 24 (2015) 055016 (13pp).

[5] S. J. Dyke, B. F. Spencer Jr., M. K. Sain, J. D. Carlson, Modeling and control of magnetorheological dampers for seismic response reduction, Smart Materials and Structures 5 (1996) 565-575.

[6] F. Weber, C. Boston, Clipped viscous damping with negative stiffness for semi-active cable damping, Smart Materials and Structures 20 (2011) 045007 (13pp).

[7] Y. J. Cha, J. Zhang, A. K. Agrawal, B. Dong, A. Friedman, S. J. Dyke, J. Ricles, Comparative studies of semiactive control strategies for $\mathrm{mr}$ dampers: pure simulation and real-time hybrid tests, Journal of Structural Engineering 139 (7) (2013) 1237-1248.

[8] B. F. Spencer Jr, M. K. Sain, Controlling buildings: a new frontier in feedback, Control Systems, IEEE 17 (6) (1997) 19-35. 
[9] M. H. Tso, J. Yuan, W. O. Wong, Design and experimental study of a hybrid vibration absorber for global vibration control, Engineering Structures 56 (2013) 1058-1069.

[10] S. T. Wu, Y. Y. Chiu, Y. C. Yeh, Hybrid vibration absorber with virtual passive devices, Journal of Sound and Vibration 299 (2007) 247-260.

[11] J. Høgsberg, M. L. Brodersen, Hybrid viscous damper with filtered integral force feedback control, Journal of Vibration and Control 22 (6) (2016) 1645-1656.

[12] M. L. Brodersen, J. Høgsberg, Hybrid damper with stroke amplification for damping of offshore wind turbines, Wind Energy (2016) DOI: 10.1002/we.1977.

[13] A. Preumont, Vibration control of active structures: an introduction, Vol. 179, Springer, 2011.

[14] M. Nakashima, N. Masaoka, Real-time on-line test for mdof systems, Earthquake engineering \& structural dynamics 28 (4) (1999) 393-420.

[15] A. Blakeborough, M. S. Williams, A. P. Darby, D. M. Williams, The development of real-time substructure testing, Philosophical Transactions of the Royal Society of London. Series A: Mathematical, Physical and Engineering Sciences 359 (1786) (2001) 1869-1891.

[16] J. Høgsberg, S. Krenk, Linear control strategies for damping of flexible structures, Journal of Sound and Vibration 293 (1) (2006) 59-77.

[17] R. Christenson, Y. Z. Lin, A. Emmons, B. Bass, Large-scale experimental verification of semiactive control through real-time hybrid simulation 1, Journal of Structural Engineering 134 (4) (2008) 522-534.

[18] J. Jonkman, W. Musial, Offshore code comparison collaboration (OC3) for IEA task 23 offshore wind technology and deployment, Tech. rep., National Renewable Energy Laboratory, nREL/TP-500-48191 (2010).

[19] J. A. Main, S. Krenk, Efficiency and tuning of viscous dampers on discrete systems, Journal of Sound and Vibration 286 (1-2) (2005) 97-122. 
[20] G. Ou, A. I. Ozdagli, S. J. Dyke, B. Wu, Robust integrated actuator control: experimental verification and real-time hybrid-simulation implementation, Earthquake Engineering \& Structural Dynamics 44 (3) (2015) 441-460.

[21] G. Ou, M. L. Brodersen, S. Dyke, Real time hybrid simulations case study: Vibration reduction of offshore wind turbine using hybrid controlled damper. 